ARTICLE

https://doi.org/10.1038/s41467-019-10738-4

\title{
Van der Waals negative capacitance transistors
}

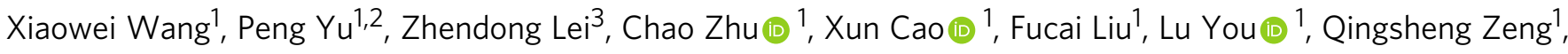 \\ Ya Deng ${ }^{1}$, Chao Zhu ${ }^{1}{ }^{1}$, Jiadong Zhou', Qundong Fu', Junling Wang $\mathbb{1}^{1}{ }^{1}$, Yizhong Huang ${ }^{1} \&$ Zheng Liu (1) 1,4,5,6
}

The Boltzmann distribution of electrons sets a fundamental barrier to lowering energy consumption in metal-oxide-semiconductor field-effect transistors (MOSFETs). Negative capacitance FET (NC-FET), as an emerging FET architecture, is promising to overcome this thermionic limit and build ultra-low-power consuming electronics. Here, we demonstrate steep-slope NC-FETs based on two-dimensional molybdenum disulfide and $\mathrm{CulnP}_{2} \mathrm{~S}_{6}$ (CIPS) van der Waals (vdW) heterostructure. The vdW NC-FET provides an average subthreshold swing (SS) less than the Boltzmann's limit for over seven decades of drain current, with a minimum SS of $28 \mathrm{mV} \mathrm{dec}^{-1}$. Negligible hysteresis is achieved in NC-FETs with the thickness of CIPS less than $20 \mathrm{~nm}$. A voltage gain of 24 is measured for vdW NC-FET logic inverter. Flexible vdW NC-FET is further demonstrated with sub- $60 \mathrm{mV} \mathrm{dec}^{-1}$ switching characteristics under the bending radius down to $3.8 \mathrm{~mm}$. These results demonstrate the great potential of vdW NC-FET for ultra-low-power and flexible applications.

\footnotetext{
${ }^{1}$ School of Materials Science and Engineering, Nanyang Technological University, Singapore 639798, Singapore. ${ }^{2}$ School of Materials Science and Engineering, Sun Yat-sen University, Guangzhou, 510275 Guangdong, China. ${ }^{3}$ NUS Graduate School for Integrative Sciences \& Engineering, National University of Singapore, Singapore 117583, Singapore. ${ }^{4}$ CINTRA CNRS/NTU/THALES, UMI 3288, Research Techno Plaza, Singapore 637553, Singapore. ${ }^{5}$ Centre for Micro-/Nano-electronics (NOVITAS), School of Electrical and Electronic Engineering, Nanyang Technological University, Singapore 639798, Singapore. ${ }^{6}$ Environmental Chemistry and Materials Centre, Nanyang Environment and Water Research Institute, Singapore 637141, Singapore. Correspondence and requests for materials should be addressed to P.Y. (email: yupengsg@gmail.com) or to Z.L. (email: z.liu@ntu.edu.sg)
} 
B oltzmann's limit is inevitable for traditional metal-oxidesemiconductor field-effect transistors (MOSFETs), which thermodynamically defines the lower limit of the subthreshold swing (SS) of $60 \mathrm{mV} \mathrm{dec}^{-1}$ at room temperature ${ }^{1,2}$, and subsequently, sets a barrier to further reduce the power consumption. Therefore, energy efficient device concepts based on scalable materials become the key to meet the great demanding in ultra-low-power applications, such as Internet-of-Things and wearable computing electronics. Negative capacitance (NC) fieldeffect transistor (NC-FET) has been proposed as one of the promising candidates beyond complementary metal-oxidesemiconductor (CMOS) device that may overcome the thermionic limit of $60 \mathrm{mV} \mathrm{dec}^{-1}$ by the internal amplification of gate voltage through ferroelectric materials ${ }^{3-7}$. Owing to their atomically thin body nature, two-dimensional (2D) transition metal dichalcogenides (TMDs) have been demonstrated to provide superior immunity to short-channel-effects ${ }^{8-11}$ and suggested to achieve steep subthreshold slope over a wide voltage range for the NC-FET ${ }^{12-14}$. NC-FETs have been reported with TMDs as channel material and ferroelectric hafnium zirconium oxide $(\mathrm{HZO})^{15-17}$ or polymer ${ }^{14,18}$ as ferroelectric gate. Comparing to the bulk ferroelectrics, layered ferroelectrics with atomically smooth surface may offer great performance and high reliability for NC-FETs by minimizing the dangling bonds and charged impurities induced interface traps ${ }^{19-21}$. A few 2D layered materials have been theoretically predicted or experimentally confirmed as ferroelectrics ${ }^{22-25}$. Among them, $\mathrm{CuInP}_{2} \mathrm{~S}_{6}$ (CIPS) has been shown with switchable polarization down to $4 \mathrm{~nm}$ at room temperature ${ }^{24}$. At the time of writing of this paper, Si et al. ${ }^{26}$ reported the ferroelectric FET based on $\mathrm{MoS}_{2}$ and CIPS heterostructure, but the sub-thermionic switching was not demonstrated due to the suspended gate structure.

In this work, we demonstrate the room temperature sub-60

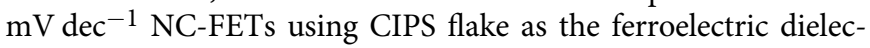
tric and atom-thin semiconductor as the channel. The average SS is less than $60 \mathrm{mV} \mathrm{dec}^{-1}$ for over seven decades of drain current and the minimum SS is down to $28 \mathrm{mV} \mathrm{dec}^{-1}$. The hysteresis window in vdW NC-FET is suppressed by decreasing the thickness of CIPS or incorporating a thin hexagonal boron nitride (h$\mathrm{BN})$ layer into the NC gate stack. High-gain logic inverter based on vdW NC-FET is built. Bending tests show that sub- $60 \mathrm{mV}$ dec ${ }^{-1}$ SS can be retained and hysteresis alleviated for vdW NC-FET on polyester substrate under a bending radius down to $3.8 \mathrm{~mm}$, benefiting from the intrinsic high flexibility and stretchability of 2D materials.

\section{Results}

Device design and heterostructure characterization. The schematic structure of a CIPS/ $\mathrm{MoS}_{2}$ vdW NC-FET is shown in Fig. 1a, consisting of a few-layer $\mathrm{MoS}_{2}$ as the channel material, CIPS flake and $285 \mathrm{~nm}$-thick $\mathrm{SiO}_{2}$ as the top $\mathrm{NC}$ and back MOS gate dielectric, respectively, heavily doped silicon substrate as the MOS gate electrode and $\mathrm{Cr} / \mathrm{Au}$ as the $\mathrm{NC}$ gate electrode and source/ drain contacts (the detailed gate-stack structure of a typical CIPS/ $\mathrm{MoS}_{2}$ vdW NC-FET is provided in Supplementary Fig. 1). The top-view layout of the devices is given in the false-color scanning electron microscopy (SEM) image (Fig. 1b), where the channel length is slightly larger than the top gate length.

The cross-sectional transmission electron microscope (TEM) image in Fig. 1c shows the layered structure of a typical vdW ferroelectric/semiconductor heterostructure created with atomically flat CIPS and $\mathrm{MoS}_{2}$ flakes via the dry transfer process ${ }^{27}$ (Supplementary Note 1 and Supplementary Fig. 2). An atomically sharp and chemically clean interface is achieved between the vdW ferroelectric and semiconductor. The high interface quality would enable the vdW NC-FET with good performance since the NC effect is strongly correlated to interface ferroelectric domain switching. Energy-dispersive X-ray spectrometry (EDS) elemental map presented in Fig. 1c confirms the uniform distribution of $\mathrm{Mo}, \mathrm{S}, \mathrm{Cu}$, In, and P. The ferroelectricity of CIPS was investigated using piezoresponse force microscopy (PFM) under dual AC resonance tracking (DART) mode (details about the DART mode PFM are provide in Supplementary Note 2 and Supplementary Fig. 3 and 4). The bright and dark regions arising from upward and downward polarizations of CIPS are clearly observed in both amplitude (Fig. 1d) and phase (Fig. 1e) images of local piezoresponse. The off-field PFM amplitude and phase hysteresis loops at individual point during the switching process are presented in Fig. If (see Supplementary Fig. 5 for the raw data). The butterfly loop in PFM amplitude and $180^{\circ}$ phase change in the nearly square PFM phase loop confirm the good ferroelectric switching nature of CIPS. Single frequency PFM (Supplementary Fig. 6), polarization switching (Supplementary Fig. 7) and polarization versus voltage $(P-V)$ hysteresis loop measurements (Supplementary Fig. 8) were also conducted to demonstrate the room temperature ferroelectricity in CIPS. Layer number of $\mathrm{MoS}_{2}$ was determined by Raman spectroscopy (Supplementary Figs. 9 and 10).

Electrical measurement of vdW NC-FETs. The room temperature electrical performance of a four-layer $\mathrm{MoS}_{2}$ device with the CIPS thickness of $51 \mathrm{~nm}$, channel length of $5.7 \mu \mathrm{m}$ and width of $5.1 \mu \mathrm{m}$ and top gate length $3.2 \mu \mathrm{m}$ is shown in Fig. $2 \mathrm{a}-\mathrm{f}$. Figure $2 \mathrm{a}$ shows the schematic of back-gate measurement configuration with $285 \mathrm{~nm} \mathrm{SiO}_{2}$ as the gate dielectric and top gate floating. The $I_{\mathrm{ds}}-V_{\mathrm{bg}}$ characteristics in Fig. 2b show a typical n-type behavior with an on/off ratio of $10^{7}$. The clockwise hysteresis between the forward and reverse sweeps can be attributed mainly to charge trapping at the interface of $\mathrm{SiO}_{2} / \mathrm{MoS}_{2}$ and $\mathrm{MOS}_{2} /$ adsorbates 28 and is suppressed to half of the original value through vacuum annealing (Supplementary Fig. 11). As shown in Fig. 2c, the minimum SS of $\mathrm{MoS}_{2}$ MOSFET is derived to be $1.698 \mathrm{~V} \mathrm{dec}^{-1}$ for forward sweep and $0.731 \mathrm{~V} \mathrm{dec}^{-1}$ for reverse sweep according to $\mathrm{SS}=\partial V_{\mathrm{bg}} /\left(\log I_{\mathrm{ds}}\right)$. Both values are far above the thermionic limit at room temperature due to the poor gate efficiency. Contrastingly, for top-gate measurement with CIPS as the ferroelectric gate insulator, so called NC-FET as illustrated in Fig. 2d, the $I_{\mathrm{ds}}-V_{\mathrm{tg}}$ characteristics (linear scale plot of the $I_{\mathrm{ds}}-V_{\mathrm{tg}}$ curve is provided in Supplementary Fig. 12) exhibit a sustained sub-60 $\mathrm{mV} \mathrm{dec}{ }^{-1}$ switching via the internal gate voltage amplification in NC capacitor. The conversion from clockwise hysteresis loop (Fig. 2b) to anticlockwise one (Fig. 2e) by top gating is a result of ferroelectric nature of CIPS and the hysteresis is found to be suppressed by reducing the $V_{\mathrm{tg}}$ sweep speed (Supplementary Fig. 13). Compared with $\mathrm{SiO}_{2}$ gating, the off-state current is significantly reduced in NC-FET due to the trap-free vdW interface between $\mathrm{MoS}_{2}$ and CIPS, and the same on/off ratio is achieved despite the limited on-state current by ungated channel segments. SS extracted from the transfer characteristics of NCFET falls below the thermionic limit for both forward and reverse sweeps, with a minimum of 39 and $28 \mathrm{mV} \mathrm{dec}^{-1}$, respectively. Incompletely compensated upward polarization in CIPS due to the low hole concentration in $\mathrm{MoS}_{2}$ leads to a larger SS for forward sweep ${ }^{18}$. The average SS for reverse sweep is $<60 \mathrm{mV} \mathrm{dec}^{-1}$ for over five decades of drain current. The effectiveness of NC effect in vdW NC-FETs is also supported by the observed draininduced-barrier-rising effect and negative-differential-resistance characteristics (Supplementary Note 3 and Supplementary Fig. 14), which are distinctive features not seen in the conventional MOSFETs. P-type vdW NC-FETs with sub-60 $\mathrm{mV} \mathrm{dec}^{-1}$ 
a

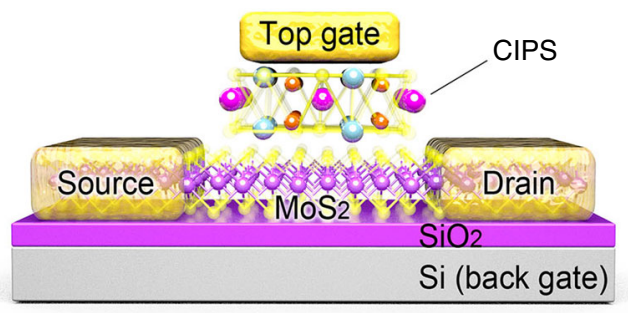

d

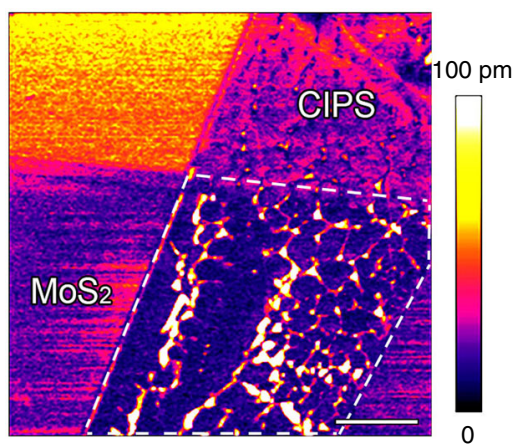

b

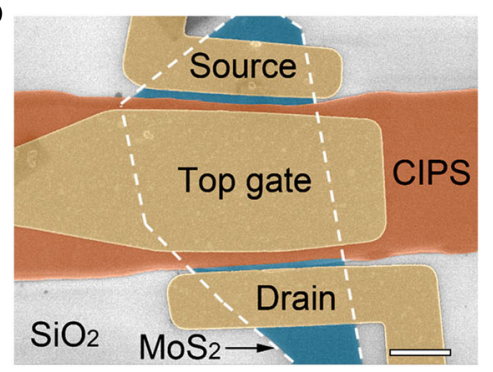

e

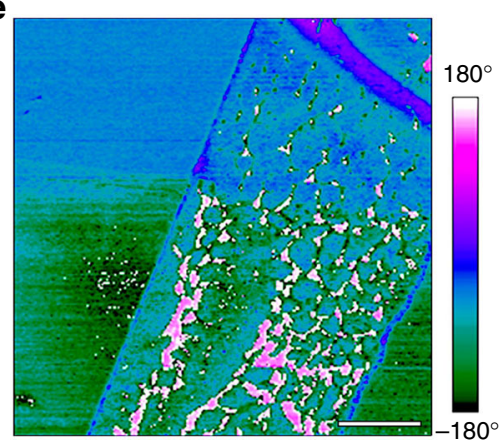

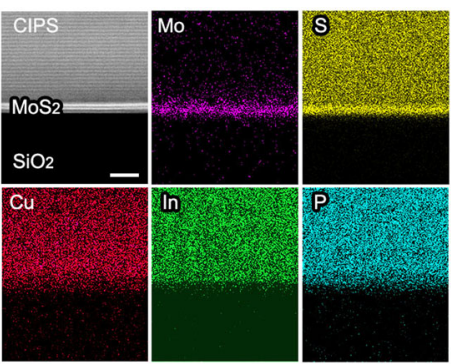

f

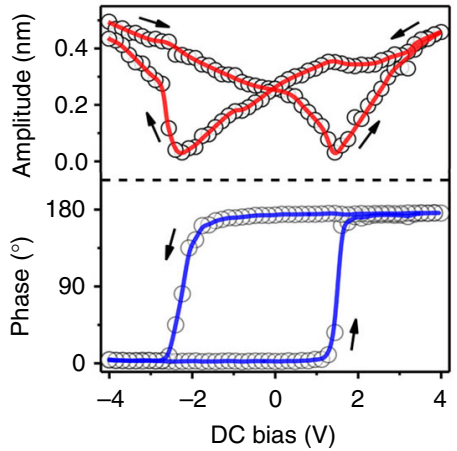

Fig. 1 CIPS/MoS 2 vdW heterostructure and NC-FET. a, b Schematic diagram (a) and False-color SEM image (b) of a CIPS/MoS 2 vdW NC-FET. Scale bar, 2 $\mu \mathrm{m}$. c Cross-sectional high-resolution TEM image of a vertically stacked CIPS/MoS 2 heterostructure on SiO $2 / \mathrm{Si}$ substrate and corresponding EDS elemental map showing the distribution of Mo, S, Cu, In, and P. Scale bar, $5 \mathrm{~nm}$. d, e PFM amplitude (d) and phase (e) of a CIPS/MoS 2 vdW heterostructure. The CIPS/MoS 2 stacked region is enclosed by dashed lines in (d). Scale bar, $2 \mu \mathrm{m}$. f The off-field PFM amplitude (top) and phase (bottom) hysteresis loops during the switching process for CIPS flake

SS were also demonstrated with electrically doped $\mathrm{WSe}_{2}$ as the channel material (Supplementary Fig. 15).

We then examine the impact of back-gate biasing on the topgate transfer characteristics of NC-FET. We found that the ferroelectric hysteresis can be suppressed by positive $V_{\text {bg }}$ (Fig. 2g) while the SS is slightly improved by negative $V_{\mathrm{bg}}$ (inset in Fig. $2 \mathrm{~g}$ ). We ascribe these effects to the back-gate modulation of CIPS capacitance $\left(C_{\mathrm{CIPS}}\right)$. A vdW NC-FET can be represented as an underlying 2D FET in series with a ferroelectric CIPS capacitor. Therefore, the internal gate voltage amplification gain is derived as $A_{\mathrm{V}}=\left|C_{\mathrm{CIPS}}\right| /\left(C_{\mathrm{CIPS}} \mid-C_{\text {int }}\right)$, where $C_{\text {int }}$ is the top-gate capacitance of underlying $2 \mathrm{D}$ FET. Then the SS of the vdW NCFET can be expressed as $\mathrm{SS}_{\mathrm{NCFET}}=\mathrm{SS}_{2 \mathrm{DFET}} / A_{\mathrm{V}}$. To obtain a large $A_{\mathrm{V}}$ and small SS, $C_{\text {int }}$ should be very close to $\left|C_{\mathrm{CIPS}}\right|$. However, in order to avoid hysteresis, $C_{\text {int }}$ must be smaller than | $C_{\text {CIPS }}{ }^{29}$. The increase of underlying $\mathrm{MoS}_{2}$ FET channel charge by applying a positive $V_{\mathrm{bg}}$ leads to an increase in $\left|C_{\mathrm{CIPS}}\right|$, resulting in a reduced hysteresis and increased SS for NC-FET.

Device architecture optimization. Optimized vdW NC-FETs were fabricated to achieve steep switching and reduce the hysteresis by controlling the thickness of ferroelectric CIPS layer. Figure $2 \mathrm{~h}$ shows the transfer characteristics of vdW NC-FETs with 23.0, 15.4, and $13.3 \mathrm{~nm}$ CIPS $\left(I_{\mathrm{ds}}-V_{\mathrm{tg}}\right.$ characteristics of NCFETs with 29.0 and $20.0 \mathrm{~nm}$ CIPS are provided in Supplementary Fig. 16). A minimum SS of $41.8 \mathrm{mV} \mathrm{dec}^{-1}$ for reverse sweep with a hysteresis of $70 \mathrm{mV}$ at $I_{\mathrm{ds}}=10 \mathrm{pA}$ is achieved for $23.0 \mathrm{~nm}$ CIPS, less than the one of $453 \mathrm{mV}$ for $51.0 \mathrm{~nm}$ CIPS shown in Fig. 2e. The average SS during reverse sweep is less than $60 \mathrm{mV} \mathrm{dec}^{-1}$ for over seven decades of drain current, which is three orders of magnitude greater than that of TMD NC-FETs with bulk ferroelectric $^{15-17}$ (Supplementary Table 1). The great transistor performance can be attributed to the strong $\mathrm{NC}$ effect due to the trap-free CIPS/ $\mathrm{MoS}_{2}$ interface in vdW NC-FET. As the thickness of CIPS decreases, its drain current range for $\mathrm{SS}<60 \mathrm{mV} \mathrm{dec}-1$ (over five decades for $15.4 \mathrm{~nm}$ CIPS and less than one decade for $13.3 \mathrm{~nm}$ CIPS) deteriorates. Nevertheless, the hysteresis of vdW NC-FET with a $13.3 \mathrm{~nm}$ CIPS is suppressed to a negligible value $(3.4 \mathrm{mV})$ while the minimum SS $\left(20.6 \mathrm{mV} \mathrm{dec}^{-1}\right.$ for forward sweep and $48.6 \mathrm{mV} \mathrm{dec}^{-1}$ for reverse sweep) are still less than the thermionic limit. The effect of CIPS thickness on hysteresis and SS can be explained by the size effects on ferroelectricity of CIPS $^{30}$ and capacitance matching between $\mathrm{C}_{\mathrm{CIPS}}$ and $\mathrm{C}_{\text {int }}$. Thinning CIPS leads to a decrease in the remnant ferroelectric polarization, the steepness and width of the hysteresis loops, as shown in Supplementary Fig. 17, resulting in a larger SS and smaller hysteresis. Moreover, $\left|C_{\mathrm{CIPS}}\right|$ increases with decreasing the thickness of CIPS, leading to a small gate-voltage amplification $A_{\mathrm{V}}$ and approaching to the hysteresis-free condition for NC-FET $\left(\left|C_{\text {CIPS }}\right|>C_{\text {int }}\right)$. More than $28 \mathrm{vdW}$ NC-FETs on $\mathrm{SiO}_{2} / \mathrm{Si}$ substrate have been successfully fabricated with CIPS thickness from 13 to $80 \mathrm{~nm}$, and CIPS thickness dependence of SS and hysteresis are summarized in Fig. 2i. Most devices (21 devices) exhibit $\mathrm{SS}<60$ $\mathrm{mV} \mathrm{dec}^{-1}$ at room temperature and the main trend of measured SS is captured well by our model simulations (details are provided in Supplementary Note 4 and Supplementary Figure 18-23). According to the simulation results, the design space for sub-60 $\mathrm{mV} \mathrm{dec}-1$ SS and hysteresis-free operation in a vdW NC-FET gated by a CIPS layer is limited to $t_{\mathrm{CIPS}}<21 \mathrm{~nm}$, and the SS can only be designed to $57 \mathrm{mV} \mathrm{dec}^{-1}$ to avoid hysteresis.

In order to further optimize the performance of NC-FET, thin h-BN layers were integrated to the top-gate stack for capacitance matching and gate leakage current reduction, as illustrated in Fig. 3a. From the simplified equivalent capacitance network in Fig. 3b, incorporating a $7.5 \mathrm{~nm} \mathrm{BN}$ layer (see Supplementary Fig. 24 for atomic force microscopy (AFM) characterization) into the top-gate stack can improve the 
a

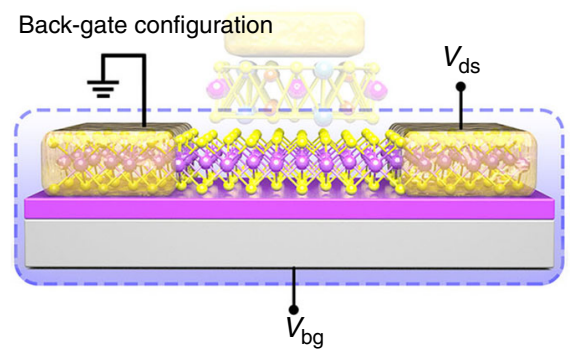

d

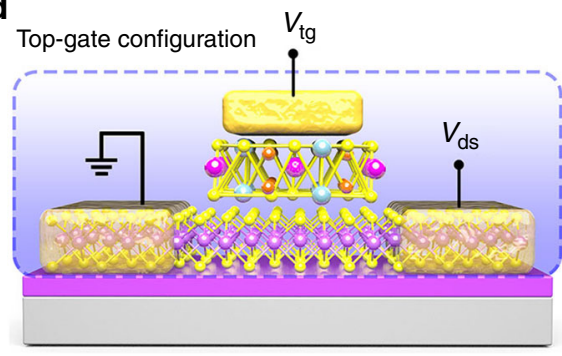

g

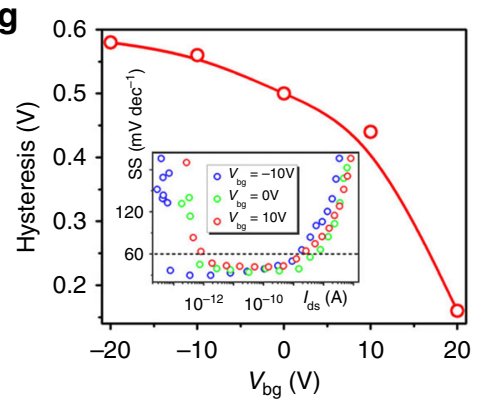

b
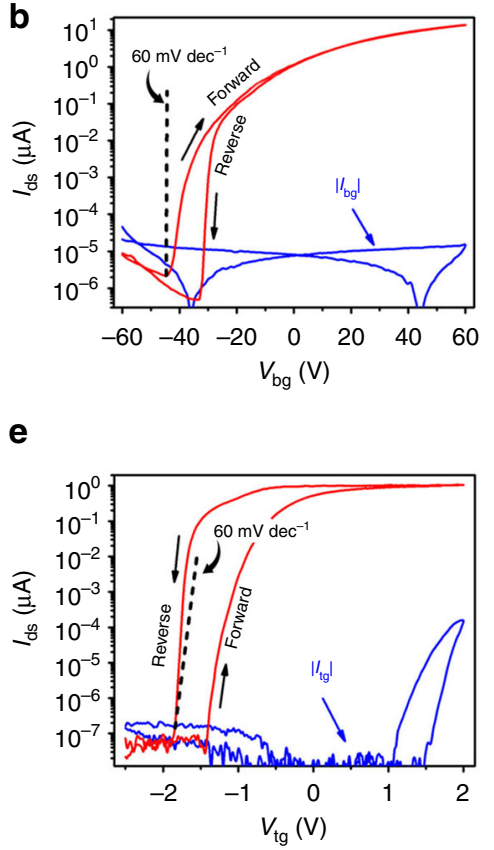

h

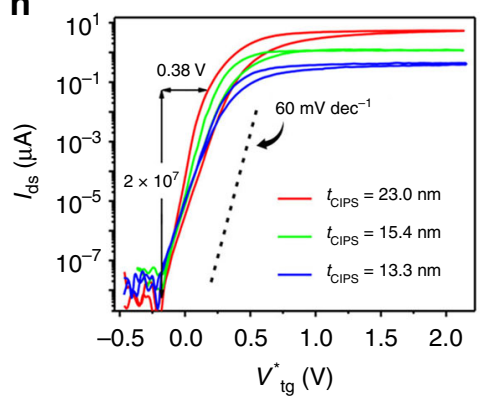

C

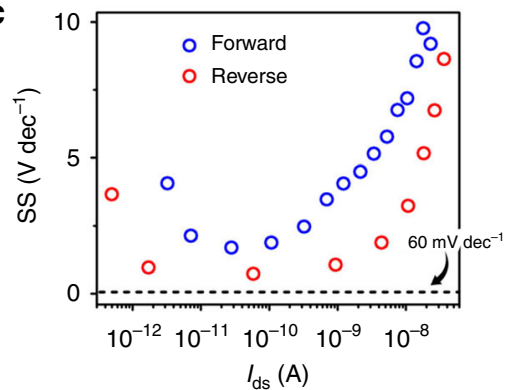

f

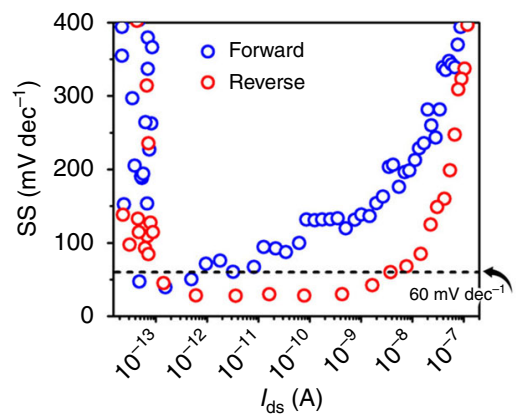

i

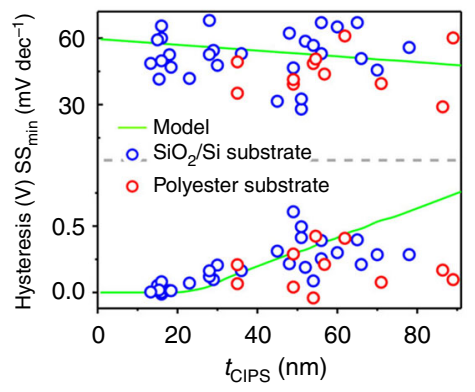

Fig. 2 Room temperature electric characterization of CIPS/MoS 2 vdW NC-FETs. a Schematics of the characterization configuration for back-gate measurements. b, c Back-gate $I_{\mathrm{ds}}-V_{\mathrm{bg}}$ characteristics (red) and leakage current (blue) $(\mathbf{b})$ and $\mathrm{SS}-I_{\mathrm{ds}}$ characteristics (c) of a CIPS/MoS $2 \mathrm{NC}-\mathrm{FET}$. $V_{\mathrm{ds}}=$ $0.5 \mathrm{~V}$. d Schematics of the characterization configuration for top-gate measurements. e, f Top-gate $I_{\mathrm{ds}}-V_{\mathrm{tg}}$ characteristics (red) and leakage current (blue) $(\mathbf{e})$ and SS $-I_{\mathrm{ds}}$ characteristics (f) of the same device as in (b). $\mathbf{g}$ Ferroelectric hysteresis dependence on $V_{\mathrm{bg}}$. Inset: SS extracted from the top-gate $I_{\mathrm{ds}}-V_{\mathrm{tg}}$ characteristics at various $V_{\mathrm{bg}}$. $\mathbf{h}$ Top-gate transfer characteristics of vdW NC-FETs with different thickness of CIPS. $V_{\text {tg }}^{\star}=V_{\mathrm{tg}}-V_{\text {th }}$, where $V_{\text {th }}$ is the threshold voltage measured with top gate. i CIPS thickness dependence of SS (top) and hysteresis width (bottom). Symbol, experimental data; Line, simulation

capacitance matching between CIPS and underlying 2D FET by reducing $C_{\text {int }}$, and thus leading to a suppression of hysteresis from 607 to $98 \mathrm{mV}$ and negligible degradation of SS for reverse sweep, as shown in Fig. 3c, d, respectively. At the same time, the gate leakage current is reduced by more than 3 orders as shown in Fig. 3e. The design space for vdW NC-FET with BN interfacial layer was also explored using our compact model. The color area represents the design space for vdW NC-FET with non-hysteresis and sub- $60 \mathrm{mV} \mathrm{dec}^{-1} \mathrm{SS}$. Obviously, the design space is considerably enlarged by integrating the thin $\mathrm{BN}$ into the gate stack. The simulation results show a hysteresisfree characteristic for vdW NC-FET with $7.5 \mathrm{~nm} \mathrm{BN}$ and $48 \mathrm{~nm}$ CIPS, while a hysteresis of $98 \mathrm{mV}$ is observed in Fig. 3c. The deviation between experimental and model results can be explained by the non-uniformity in potential and charge at the CIPS/BN interface due to the absence of interfacial metal layer in the real device ${ }^{31}$.

VdW NC-FET inverters. A logic inverter was fabricated to evaluate the feasibility of vdW NC-FET for low-power applications. As shown in the schematic (Fig. 4a, b), the logic inverter was constructed with two CIPS/MoS 2 vdW NC-FETs connected in series, serving as the pull-up load and pull-down driver, respectively. The pull-up load was realized by directly connecting the top gate of a NC-FET to the common source electrode. A typical vdW NC-FET inverter with $W / L=5.4 / 4.0$ for load NC-FET and $W / L=2.4 / 5.5$ for driver NC-FET, is shown in the false-color SEM image of Fig. 4c, where $W$ and $L$ denote the width and length of the transistor channels, respectively. Figure $4 \mathrm{~d}$ presents the voltage transfer curves, plot of input $\left(V_{\text {IN }}\right)$ versus output voltage $\left(V_{\text {OUT }}\right)$, of vdW NC-FET inverter under various supply voltages $\left(V_{\mathrm{DD}}\right)$. Signal inversions are clearly observed with high $V_{\text {OUT }}$ at low $V_{\text {IN }}$ even though the $V_{\mathrm{DD}}$ is down to $0.1 \mathrm{~V}$ for both forward and reverse sweeps. Comparing the $V_{\text {OUT }}$ versus $V_{\text {IN }}$, a maximum voltage gain as $\sim 24$ can be obtained for $V_{\mathrm{DD}}=1.5 \mathrm{~V}$, which is considerably higher in comparison with TMDs based MOS inverters ${ }^{32-34}$. The noise margins of the inverter, $\mathrm{NM}_{\mathrm{L}}=0.406 V_{\mathrm{DD}}$ and $\mathrm{NM}_{\mathrm{H}}=0.493 V_{\mathrm{DD}}$ ((see Supplementary Note 5 and Supplementary Fig. 25 for determination of the noise margins), approach the idea noise margin $\left(0.5 V_{\mathrm{DD}}\right)$, indicating that the vdW NC-FET inverter is 
a

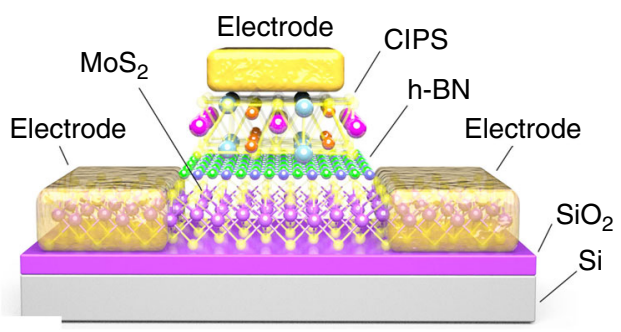

\section{C}

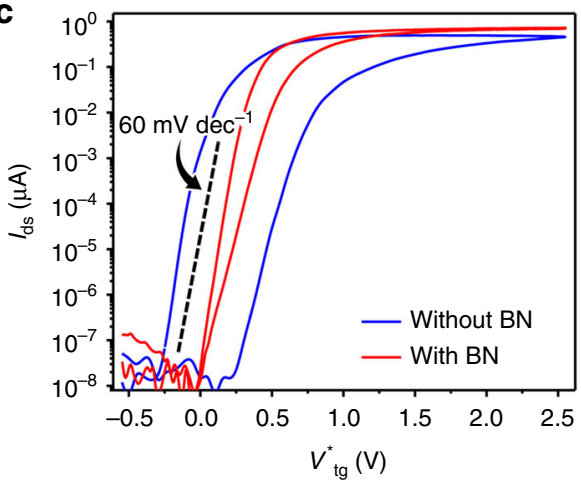

e

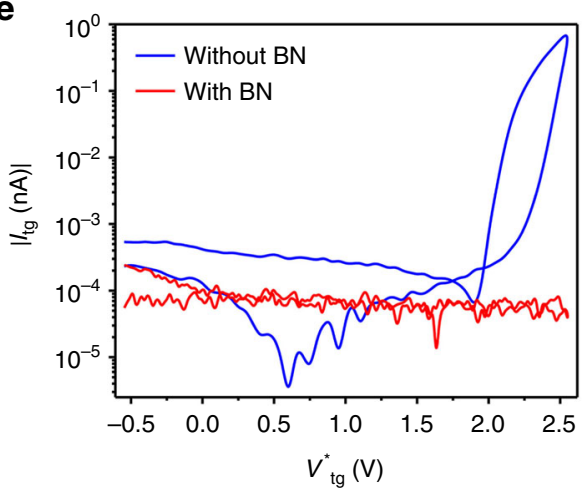

b

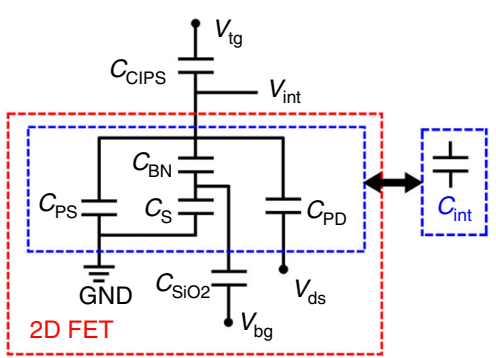

d

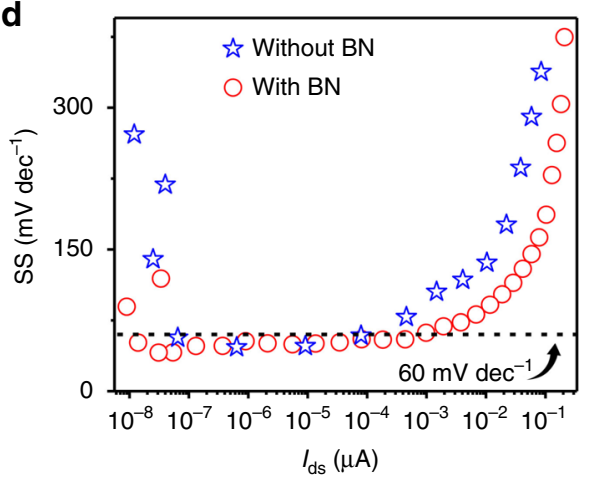

f

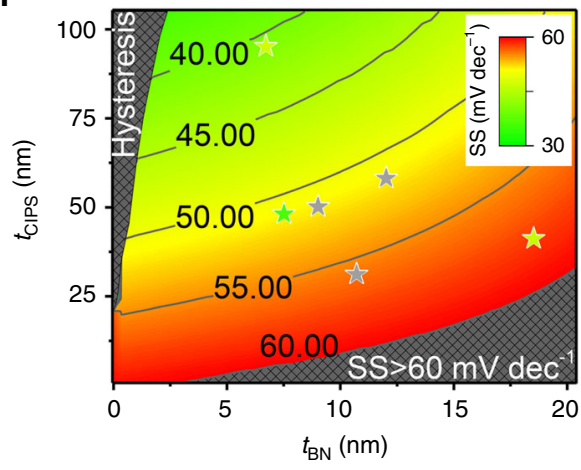

Fig. 3 Electric characterization of CIPS/BN/MoS 2 vdW NC-FETs. a, b Schematic diagram (a) and equivalent capacitor network (b) of a CIPS/BN/MoS 2 vdW NC-FET. c Top-gate transfer characteristics of vdW NC-FETs with and without interfacial h-BN layer. $V_{\text {tg }}^{\star}=V_{\text {tg }}-V_{\text {th }}$. Thickness of CIPS in CIPS/MoS 2 NC-FET is $49 \mathrm{~nm}$ and in CIPS/BN/MoS 2 NC-FET is $48 \mathrm{~nm}$. The thickness of BN layer is $7.5 \mathrm{~nm}$. d, e Top-gate SS-l $I_{\text {ds }}$ characteristics for reverse sweep (d) and leakage current (e) of CIPS/MoS 2 and CIPS/BN/MoS 2 vdW NC-FETs. f Contour plot of simulated SS as a function of thickness of CIPS ( $t_{\mathrm{CIPS}}$ ) and BN layer $\left(t_{\mathrm{BN}}\right)$ at $V_{\mathrm{ds}}=0.5 \mathrm{~V}$ and $V_{\mathrm{bg}}=0 \mathrm{~V}$. Symbol, experimental data

highly immune to electrical noise from the environment and very desirable for integration into multi-stage logic circuits, despite a hysteresis of $380 \mathrm{mV}$ induced by the poor capacitance matching and the intrinsic negative-differential-resistance effect in NC-FET.

VdW NC-FETs on flexible substrate. A wide range of flexible electronic devices are typically powered by energy harvesting sources, it is necessary to demonstrate the scalability of vdW NCFETs in flexible electronic applications to minimize the energy consumption. The layered structures of CIPS and TMDs offer a good mechanical flexibility for vdW NC-FET. Figure 5a, b shows the structure and photograph of a flexible $\mathrm{MoS}_{2}$ NC-FET with a pure CIPS dielectric layer atop a 130 um thick polyester substrate, respectively. The vdW NC-FET on flexible substrate exhibits a similar performance with an anticlockwise hysteresis loop and sustained sub-60 mV dec ${ }^{-1}$ switching, as shown in Fig. $5 \mathrm{c}$ (see Supplementary Figs. 26 and 27 for more vdW NC-FETs on flexible substrate). In order to investigate the stability of the device performance under static tensile strain, electrical characteristics were recorded with flexible NC-FETs under various bending curvature radius. Figure $5 \mathrm{~d}$ presents the transfer characteristics of a vdW NCFET measured at tensile bending states with bending radius $\left(R_{\mathrm{b}}\right)$ from 86.4 to $3.8 \mathrm{~mm}$ (see Supplementary Note 6 and Supplementary Figure 28 for determination of bending radius). The steep switching characteristic with the minimum SS less than $60 \mathrm{mV} \mathrm{dec}$ ${ }^{-1}$ was preserved even with $R_{\mathrm{b}}$ down to $3.8 \mathrm{~mm}$, less than minimum bending radius reported in previous organic ferroelectric devices ${ }^{35}$. The slight decrease in on-state current with decreasing $R_{\mathrm{b}}$ arises from the tensile strain induced polarization decrease in CIPS, while the increase in off-state current may result from the piezotronic effect of CIPS induced gate leakage current increase ${ }^{23}$, and $I_{\mathrm{tg}}-V_{\mathrm{tg}}$ characteristics at various bending states are provided in Supplementary Fig. 29. Surprisingly, the hysteresis window of the flexible vdW NC-FET was suppressed to $80 \mathrm{mV}$ as the bending radius decreases to $4 \mathrm{~mm}$, as shown in Fig. 5e, which is most likely due to the reduction of coercive field for stressed CIPS ${ }^{36}$. This conclusion is also supported by the thickness dependence of hysteresis shown in Fig. $2 \mathrm{i}$ and Supplementary Fig. 27, where vdW NC-FETs on flexible substrate show relatively smaller hysteresis compared to 

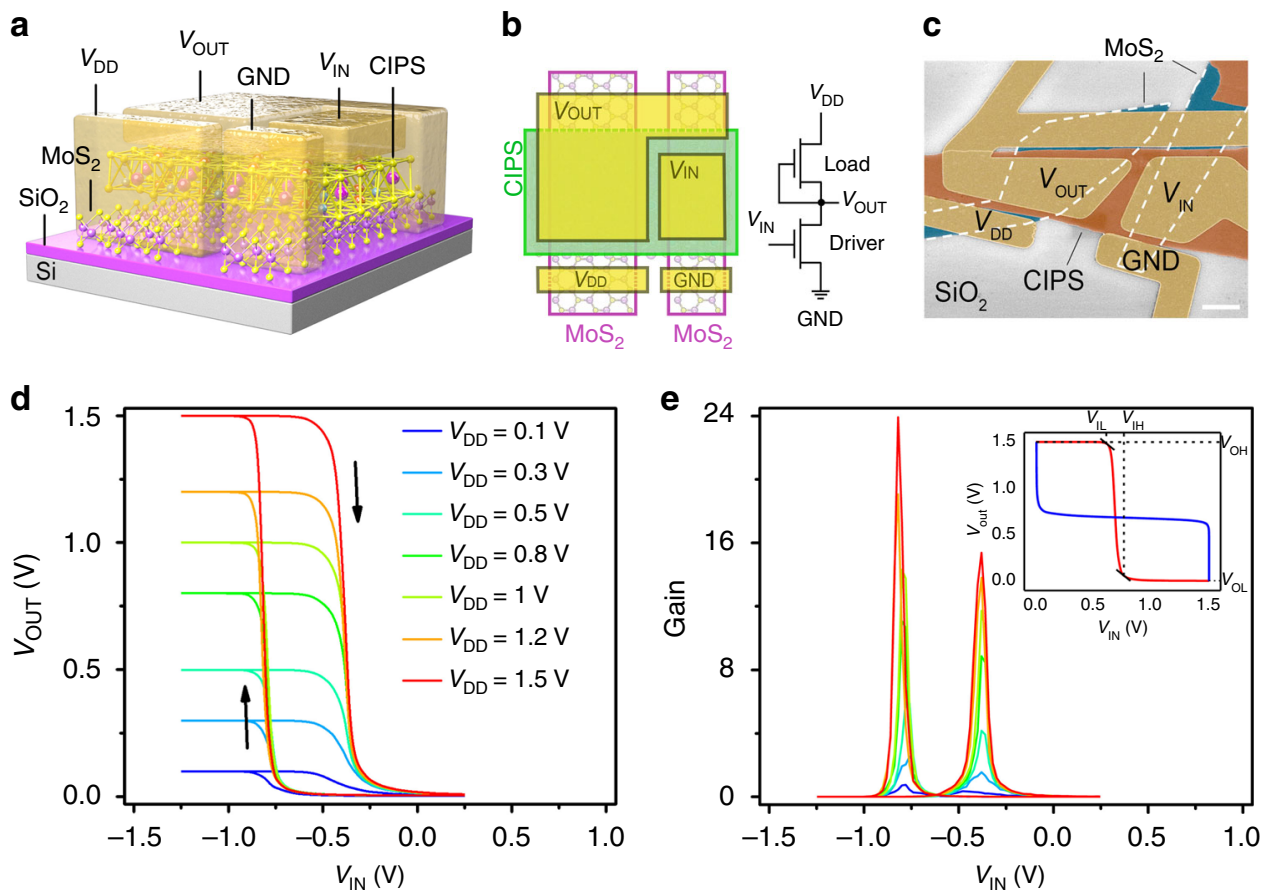

Fig. 4 Electrical performance of vdW NC-FET inverter. a-c Schematic structure (a), circuit schematic (b) and false-color SEM image (c) of a vdW NC-FET inverter. $W / L=5.4 / 4.0$ for load NC-FET and $W / L=2.4 / 5.5$ for driver NC-FET. The thickness of CIPS flake is $42 \mathrm{~nm}$. Scale bar, $2 \mu \mathrm{m}$. $\mathbf{d}$ Room temperature

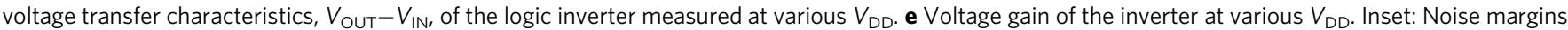
of vdW NC-FET inverter at $V_{D D}=1.5 \mathrm{~V}$

a

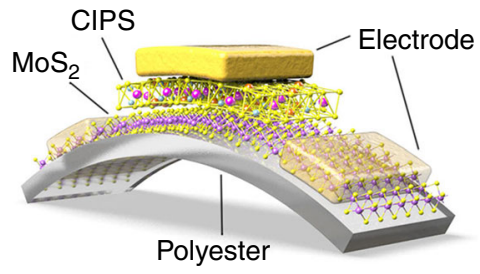

b

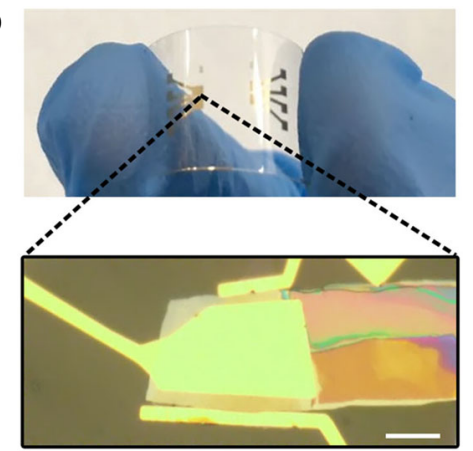

C
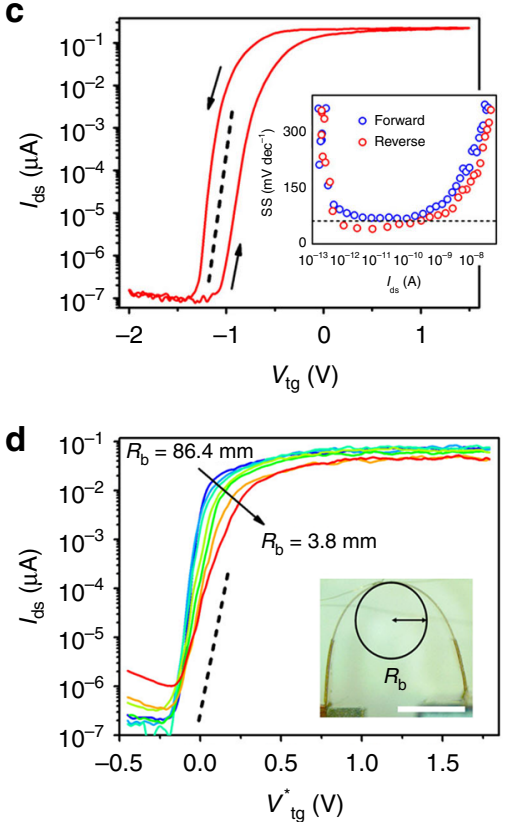
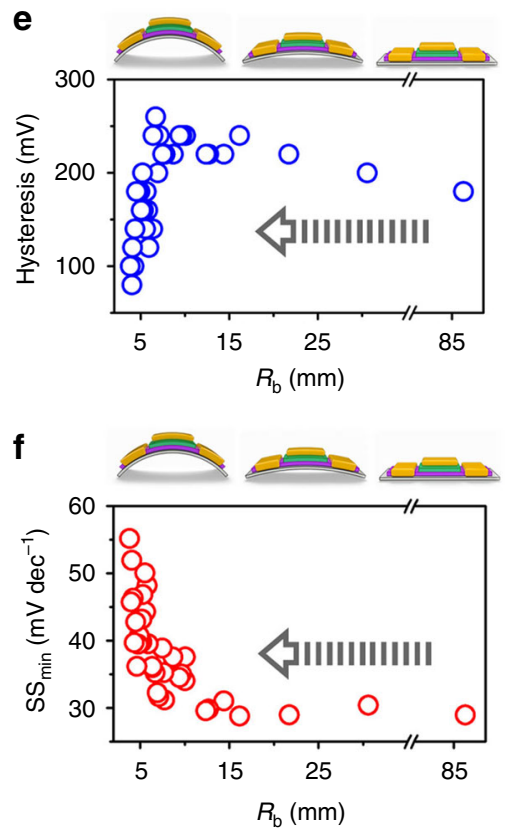

Fig. 5 VdW NC-FETs on flexible substrate. $\mathbf{a}$, b Schematic structure (a) and photograph (b) of a CIPS/MoS 2 vdW NC-FET on a flexible polyester substrate. The thickness of CIPS flake is $86.4 \mathrm{~nm}$. Scale bar, $10 \mu \mathrm{m}$. $\mathbf{c} l_{\mathrm{ds}}-V_{\mathrm{tg}}$ characteristics of a flexible vdW NC-FET. $V_{\mathrm{ds}}=0.5 \mathrm{~V}$. Inset, SS- $I_{\mathrm{ds}}$ characteristics. d Transfer characteristics of a flexible vdW NC-FET measured at the bending states with $R_{\mathrm{b}}$ values of 86.4, 12.4, 7.5, 5.8, 4.8, 4.2, and 3.8 mm. $V_{\text {tg }}^{*}=V_{\mathrm{tg}}-V_{\mathrm{th}}$. Inset, photograph of a vdW NC-FET on stressed polyester substrate. Scale bar, $5 \mathrm{~mm}$. e, $\mathbf{f}$ The effect of bending radius on the hysteresis width (e) and SS (f) of the vdW NC-FET

devices on $\mathrm{SiO}_{2} / \mathrm{Si}$ substrate due to the residual stresses introduced during device fabrication. Figure $5 \mathrm{f}$ illustrates the extracted minimum SS of the device as a function of the bending radius. Sub-30

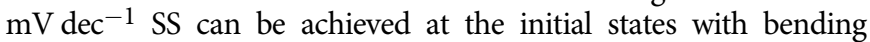
radius larger than $12 \mathrm{~mm}$ following a slight degradation of SS as $R_{\mathrm{b}}$ decreases below $10 \mathrm{~mm}$, which may result from the suppressed ferroelectric polarization due to the electrical breakdown under extreme bending condition ${ }^{37}$. However, it is clearly that all $\mathrm{SS}_{\min }$ values are better than the thermionic limit until the bending radius reaches $3.8 \mathrm{~mm}$. Bending cycle test was also carried out with 
another device and sub- $60 \mathrm{mV} \mathrm{dec}{ }^{-1}$ switching characteristics were maintained up to 500 cycles (Supplementary Note 6 and Supplementary Fig. 30). The successful demonstration of flexible NC-FET with vdW ferroelectric and semiconductor represents a strategy to meet the ultralow-power operation in the emerging wearable computing applications.

\section{Discussion}

In conclusion, NC-FETs have been successfully demonstrated using van der Waals ferroelectrics and TMDs. The adaptation to $\mathrm{vdW}$ layered semiconductor and ferroelectric enables the NCFET with high performance, originating from the clean interface between $\mathrm{MoS}_{2}$ and CIPS, and high bendability. The subthermionic switching characteristics and the observed draininduced-barrier-rising effect and negative-differential-resistance characteristics have been confirmed to be the results of NC effect of CIPS. Hysteresis is considerably reduced by integrating a thin $\mathrm{h}$-BN layer to the gate stack and negligible hysteresis is achieved in vdW NC-FETs with the thickness of CIPS less than $20 \mathrm{~nm}$. Moreover, high-gain inverter based on vdW NC-FET is built. Sub-60 $\mathrm{mV} \mathrm{dec}^{-1}$ SS can be retained and hysteresis alleviated for $\mathrm{vdW}$ NC-FETs on flexible substrate under a bending radius down to $3.8 \mathrm{~mm}$. Our work demonstrates that NC-FETs with vdW ferroelectrics and TMDs are a promising architecture for lowpower and wearable applications.

\section{Methods \\ VdW NC-FET device fabrication. High-quality single crystals of CIPS, $\mathrm{MoS}_{2}$, and $\mathrm{WSe}_{2}$ were synthesized by solid state reaction. The $2 \mathrm{D}$ flakes were all achieved by mechanical exfoliation from bulk crystals onto heavily doped silicon substrates with a $285 \mathrm{~nm} \mathrm{SiO}{ }_{2}$ layer. TMD/CIPS and TMD/BN/CIPS heterostructures were produced with a dry transfer technique as reported. $\mathrm{Cr} / \mathrm{Au}(5 \mathrm{~nm} / 80 \mathrm{~nm})$ electrodes were defined using standard e-beam lithography (EBL) process followed by metal thermal evaporation and lift-off process. Ten-nanometers Au film was used as a discharge layer for EBL processes on polyester substrate.}

Characterizations. Layer number of $\mathrm{MoS}_{2}$ and WSe $\mathrm{W}_{2}$ were identified by optical microscopy and micro-Raman spectroscopy (Witec) with a $532 \mathrm{~nm}$ laser. Atomic force microscopy (AFM, Bruker Dimension Icon or Asylum Research Cypher S) in a tapping mode was used to characterize the morphology of the CIPS and device. PFM measurements were carried out on a commercial atomic force microscope (Asylum Research Cypher S) under DART mode. Off-field hysteresis loops were obtained by recording the piezoresponse amplitude and phase 1 signals after individual DC bias was turned off. Electrical transport properties of vdW NC-FETs on $\mathrm{SiO}_{2} / \mathrm{Si}$ substrate were measured with an Agilent B1500A Semiconductor Device Parameter Analyzer in a vacuum chamber of $10^{-2}$ torr. For the bending test, a micro translation stage was used to hold the flexible substrate on both sides and a manual handle to vary the distance between the two ends of the flexible substrate to control the bending curvature. Bending radius was estimated from the optical image of the strained substrate. The electrical properties of flexible NC-FETs at different bending states were recorded with Agilent B1500A in an air environment.

Simulation. A vdW NC-FET was treated as an underlying 2D FET in series with a ferroelectric capacitor. The transfer characteristics of 2D FET were obtained by solving the Poisson and drift-diffusion equation. Steady-state Landau-Khalatnikov equation was employed to model ferroelectric CIPS capacitor.

\section{Data availability}

The data that support the findings of this study are available from the corresponding author upon reasonable request.

Received: 3 February 2019 Accepted: 17 May 2019

Published online: 10 July 2019

\section{References}

1. Taur, Y. \& Ning, T. H. Fundamentals of Modern VLSI Devices. (Cambridge University Press, New York, USA, 1998).
2. Chandrakasan, A. P. \& Brodersen, R. W. Minimizing power-consumption in digital Cmos circuits. Proc. IEEE 83, 498-523 (1995).

3. Salahuddin, S. \& Dattat, S. Use of negative capacitance to provide voltage amplification for low power nanoscale devices. Nano Lett. 8, 405-410 (2008).

4. Zhirnov, V. V. \& Cavin, R. K. Negative capacitance to the rescue? Nat. Nanotech. 3, 77-78 (2008).

5. Kobayashi, M. \& Hiramoto, T. Device design guideline for steep slope ferroelectric FET using negative capacitance in sub- $0.2 \mathrm{~V}$ operation: operation speed, material requirement and energy efficiency. VLSI Tech. Symp, 212-213 (2015).

6. $\mathrm{Li}, \mathrm{X}$. Q. et al. Enabling energy-efficient nonvolatile computing with negative capacitance FET. IEEE Trans. Electron. Dev. 64, 3452-3458 (2017).

7. Dutta, T. et al. Performance evaluation of 7-nm node negative capacitance FinFET-based SRAM. IEEE Electron. Dev. Lett. 38, 1161-1164 (2017).

8. Radisavljevic, B., Radenovic, A., Brivio, J., Giacometti, V. \& Kis, A. Single-layer $\mathrm{MoS}_{2}$ transistors. Nat. Nanotech. 6, 147-150 (2011).

9. Desai, S. B. et al. $\mathrm{MoS}_{2}$ transistors with 1-nanometer gate lengths. Science 354, 99-102 (2016).

10. Liu, L., Lu, Y. \& Guo, J. On monolayer $\mathrm{MoS}_{2}$ field-effect transistors at the scaling limit. IEEE Trans. Electron. Dev. 60, 4133-4139 (2013).

11. Chhowalla, M., Jena, D. \& Zhang, H. Two-dimensional semiconductors for transistors. Nat. Rev. Mater. 1, 16052 (2016).

12. Ota, H., Migita, S., Hattori, J., Fukuda, K. \& Toriumi, A. Structural advantages of silicon-on-insulator FETs over FinFETs in steep subthreshold-swing operation in ferroelectric-gate FETs. Jpn. J. Appl. Phys. 56, 04CD10 (2017).

13. Sharma, A. \& Roy, K. Design space exploration of hysteresis-free $\mathrm{HfZrO}_{\mathrm{x}}-$ based negative capacitance FETs. IEEE Electron. Dev. Lett. 38, 1165-1167 (2017).

14. McGuire, F. A., Cheng, Z. H., Price, K. \& Franklin, A. D. Sub-60 mV/decade switching in 2D negative capacitance field-effect transistors with integrated ferroelectric polymer. Appl. Phys. Lett. 109, 093101 (2016).

15. $\mathrm{Si}, \mathrm{M}$. W. et al. Steep-slope hysteresis-free negative capacitance $\mathrm{MoS}_{2}$ transistors. Nat. Nanotech. 13, 24-28 (2018).

16. McGuire, F. A. et al. Sustained sub- $60 \mathrm{mV} /$ decade switching via the negative capacitance effect in $\mathrm{MoS}_{2}$ transistors. Nano. Lett. 17, 4801-4806 (2017).

17. $\mathrm{Si}, \mathrm{M}$. W. et al. Steep-slope $\mathrm{WSe}_{2}$ negative capacitance field-effect transistor. Nano Lett. 18, 3682-3687 (2018).

18. Wang, X. D. et al. Two-dimensional negative capacitance transistor with polyvinylidene fluoride-based ferroelectric polymer gating. $n p j 2 D$ Mater. Appl. 1, 38 (2017).

19. Yusuf, M. H., Nielsen, B., Dawber, M. \& Du, X. Extrinsic and intrinsic charge trapping at the graphene/ferroelectric interface. Nano. Lett. 14, 5437-5444 (2014).

20. Han, M. G. et al. Interface-induced nonswitchable domains in ferroelectric thin films. Nat. Commun. 5, 4693 (2014).

21. Lee, H., Tseng, K. \& Su, P. Interface discrete trap induced variability for negative capacitance FinFETs. Int. Symp. VLSI Technol., Syst., Appl. 1-2 (2018).

22. Ding, W. et al. Prediction of intrinsic two-dimensional ferroelectrics in $\mathrm{In}_{2} \mathrm{Se}_{3}$ and other $\mathrm{III}_{2}-\mathrm{VI}_{3}$ van der Waals materials. Nat. Commun. 8, 14956 (2017).

23. Zhou, Y. et al. Out-of-plane piezoelectricity and ferroelectricity in layered alpha- $\mathrm{In}_{2} \mathrm{Se}_{3}$ nanoflakes. Nano. Lett. 17, 5508-5513 (2017).

24. Liu, F. C. et al. Room-temperature ferroelectricity in $\mathrm{CuInP}_{2} \mathrm{~S}_{6}$ ultrathin flakes. Nat. Commun. 7, 12357 (2016).

25. Belianinov, A. et al. $\mathrm{CuInP}_{2} \mathrm{~S}_{6}$ room temperature layered ferroelectric. Nano. Lett. 15, 3808-3814 (2015).

26. Si, M. W., Liao, P. Y., Qiu, G., Duan, Y. Q. \& Ye, P. D. D. Ferroelectric fieldeffect transistors based on $\mathrm{MoS}_{2}$ and $\mathrm{CuInP}_{2} \mathrm{~S}_{6}$ two-dimensional van der Waals heterostructure. Acs Nano 12, 6700-6705 (2018).

27. Castellanos-Gomez, A. et al. Deterministic transfer of two-dimensional materials by all-dry viscoelastic stamping. 2D Mater. 1, 011002 (2014).

28. Guo, Y. et al. Charge trapping at the $\mathrm{MoS}_{2}-\mathrm{SiO}_{2}$ interface and its effects on the characteristics of $\mathrm{MoS}_{2}$ metal-oxide-semiconductor field effect transistors. Appl. Phys. Lett. 106, 103109 (2015)

29. Yeung, C. W., Khan, A. I., Salahuddin, S. \& Hu, C. M. Device design considerations for ultra-thin body non-hysteretic negative capacitance FETs. Proc. Third Berkeley Symp. Energy Efficient Electron. Syst. (E3S), 1-2 (2013).

30. Chyasnavichyus, M. et al. Size-effect in layered ferrielectric $\mathrm{CuInP}_{2} \mathrm{~S}_{6} . A p p l$. Phys. Lett. 109, 172901 (2016).

31. Pahwa, G., Dutta, T., Agarwal, A. \& Chauhan, Y. S. Physical insights on negative capacitance transistors in nonhysteresis and hysteresis regimes: MFMIS versus MFIS structures. IEEE Trans. Electron. Dev. 65, 867-873 (2018).

32. Wachter, S., Polyushkin, D. K., Bethge, O. \& Mueller, T. A microprocessor based on a two-dimensional semiconductor. Nat. Commun. 8, 14948 (2017). 
33. Cheng, R. et al. Few-layer molybdenum disulfide transistors and circuits for high-speed flexible electronics. Nat. Commun. 5, 5143 (2014).

34. Wang, H. et al. Integrated circuits based on bilayer $\mathrm{MoS}_{2}$ transistors. Nano Lett. 12, 4674-4680 (2012).

35. Hwang, S. K., Bae, I., Kim, R. H. \& Park, C. Flexible non-volatile ferroelectric polymer memory with gate-controlled multilevel operation. Adv. Mater. 24, 5910-5914 (2012).

36. Wang, J., Xia, Y. F., Chen, L. Q. \& Shi, S. Q. Effect of strain and deadlayer on the polarization switching of ferroelectric thin film. J. Appl. Phys. 110, 114111 (2011).

37. $\mathrm{Yu}, \mathrm{H}$. et al. Flexible inorganic ferroelectric thin films for nonvolatile memory devices. Adv. Funct. Mater. 27, 1700461 (2017).

\section{Acknowledgements}

This work was supported by the Singapore National Research Foundation under NRF award number NRF-RF2013-08, Tier 2 MOE2015-T2-2-007, MOE2015-T2-2-043, MOE2017-T2-2-136, Tier 3 MOE2018-T3-1-002, and A*Star QTE programme.

\section{Author contributions}

Z.L. and X.W.W. conceived and designed the project. X.W.W. performed the AFM, PFM and SEM measurement, device fabrication, transport properties measurement, theoretical calculation and data analysis. P.Y. performed the single crystal CIPS and $\mathrm{WSe}_{2}$ growth. Y.Z.H. and X.C. prepared the cross-sectional TEM samples. C.Z. (ORCID: 0000-00016383-3665) conducted the TEM and EDS analyses. F.C.L., L.Y., and J.L.W. carried out the P-V measurement. X.W.W. and Z.L. summarized the manuscript. Z.D.L., Q.S.Z., Y.D., C.Z. (ORCID: 0000-0002-1589-855X), J.D.Z., and Q.D.F. discussed and commented on the manuscript.

\section{Additional information}

Supplementary Information accompanies this paper at https://doi.org/10.1038/s41467019-10738-4.

Competing interests: The authors declare no competing interests.

Reprints and permission information is available online at http://npg.nature.com/ reprintsandpermissions/

Peer review information: Nature Communications thanks the anonymous reviewer(s) for their contribution to the peer review of this work.

Publisher's note: Springer Nature remains neutral with regard to jurisdictional claims in published maps and institutional affiliations.

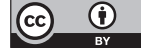

Open Access This article is licensed under a Creative Commons Attribution 4.0 International License, which permits use, sharing, adaptation, distribution and reproduction in any medium or format, as long as you give appropriate credit to the original author(s) and the source, provide a link to the Creative Commons license, and indicate if changes were made. The images or other third party material in this article are included in the article's Creative Commons license, unless indicated otherwise in a credit line to the material. If material is not included in the article's Creative Commons license and your intended use is not permitted by statutory regulation or exceeds the permitted use, you will need to obtain permission directly from the copyright holder. To view a copy of this license, visit http://creativecommons.org/ licenses/by/4.0/.

(C) The Author(s) 2019 